\title{
Estudo etnobotânico sobre plantas medicinais na comunidade de Curral Velho, Luís Correia, Piauí, Brasil
}

\author{
Jairla Lima Araujo \\ Jesus Rodrigues Lemos * \\ Universidade Federal do Piauí, Campus Ministro Reis Velloso \\ Avenida São Sebastião, 2819, CEP 64202-020, Parnaíba - PI, Brasil \\ * Autor para correspondência \\ jelemos@ib.usp.br
}

Submetido em 20/10/2014

Aceito para publicação em 18/03/2015

\section{Resumo}

A utilização das plantas como medicamento no estado do Piauí tem sido uma prática comum que vem sendo transmitida de geração a geração. Este estudo teve por objetivo analisar o uso de plantas medicinais pelos moradores da comunidade Curral Velho, município de Luís Correia, norte do Piauí, contribuindo para o registro e a preservação do conhecimento botânico tradicional da comunidade estudada e, consequentemente, do estado. O levantamento das espécies vegetais utilizadas como recurso terapêutico foi realizado por meio de entrevistas com questionário semiestruturado aplicado a 38 informantes. As plantas foram coletadas para identificação científica. Foi determinado o valor de uso (VU), fator do consenso do informante (FCI) e importância relativa (IR) das espécies. Registraram-se 62 espécies, pertencentes a 38 famílias e 57 gêneros, com destaque para a família Fabaceae. Aristolochia triangularis, Petiveria alliaceae e Stachytarpheta cayennensis apresentaram os maiores valores de uso (VU =3,0), sendo Turnera subulata a mais versátil. Dos 10 sistemas corporais identificados, os que apresentaram maior concentração de espécies medicinais estão relacionados a enfermidades mais corriqueiras como sinais gerais (inflamação, febre), doenças do aparelho respiratório e doenças do aparelho geniturinário. Esta pesquisa permitiu identificar alguns aspectos relevantes sobre o uso e o conhecimento de plantas medicinais na comunidade estudada. A diversidade de plantas medicinais conhecida e a obtenção das plantas na própria comunidade sugerem uma correlação entre uso/conhecimento de plantas medicinais e sua disponibilidade.

Palavras-chave: Importância relativa; Populações tradicionais; Valor de uso

\section{Abstract}

Ethnobotanical study on medicinal plants in the community of Curral Velho, Luís Correia, Piauí, Brazil. The use of plants as medication in the state of Piauí, Brazil, has been a common practice passed from generation to generation. This study aimed to analyze the use of medicinal plants by residents of the community Curral Velho, in the municipality of Luís Correia, northern Piauí, Brazil, contributing to register and preserve the traditional botanical knowledge of the community under study and, as a consequence, the state's. The survey of plant species used as a therapeutic resource was conducted through interviews with a semi-structured questionnaire applied to 38 informants. The plants were collected for scientific identification. Use value (UV), informant consensus factor (ICF), and relative importance (RI) of species were determined. We registered 62 species, belonging to 38 families and 57 genera, and the Fabaceae family stood out. Aristolochia triangularis, 
Petiveria alliaceae, and Stachytarpheta cayennensis had the highest use values (UV =3.0), and Turnera subulata was the most versatile. Out of the 10 bodily systems identified, those with higher concentration of medicinal species are related to the most ordinary illnesses as general signs (inflammation, fever), respiratory tract diseases, and genitourinary tract diseases. This survey enabled the identification of some relevant aspects concerning the use and knowledge of medicinal plants in the community under study. The diversity of medicinal plants known and the availability of plants in the very community suggest a correlation between use/knowledge of medicinal plants and their availability.

Key words: Relative importance; Traditional populations; Use value

\section{Introdução}

Desde a antiguidade o homem sempre esteve ligado e dependente do universo vegetal e, por meio de vivências e experiências, aprendeu a retirar seu próprio sustento, utilizando-o também para finalidades medicinais, empíricas e simbólicas (ALBUQUERQUE, 2005; LORENZI; MATOS, 2008). Franco e Barros (2006) ressaltam que o uso popular de plantas medicinais é uma arte que acompanha o ser humano desde os primórdios da civilização, sendo fundamentada no acúmulo de informações repassadas oralmente através de sucessivas gerações. Ao longo dos séculos, os produtos de origem vegetal constituíram a base para tratamento de diferentes doenças no mundo (PHILLIPS; GENTRY, 1993). Esses hábitos e costumes são tão variados entre as populações, que não se podem ter dúvidas sobre a efetiva atuação das substâncias oriundas dos vegetais (SILVA, 2003).

Esse saber das populações tradicionais vem sendo estudados pela etnobiologia, a qual aborda estudos que visam perceber o papel da natureza sob os olhares das populações locais dentro de um sistema de crenças e adaptações do homem com o meio (ALBUQUERQUE; LUCENA, 2004). Dentro da abordagem etnobiológica, um dos ramos em destaque é o da etnobotânica que estuda os conhecimentos e a utilização das plantas para os diversos fins, entre eles os medicinais (ALMEIDA; ALBUQUERQUE, 2002; ALBUQUERQUE; LUCENA, 2004; COSTA; MAYWORM, 2011).

As comunidades rurais estão intimamente ligadas ao uso de plantas medicinais, devido à sua disponibilidade, sendo, geralmente, cultivadas em hortas, quintais ou coletadas em matas (áreas de vegetação primária ou secundária), ao conhecimento acumulado de antepassados e a precariedade de assistência médica convencional, sendo as plantas, na maioria das vezes, o único recurso disponível para o tratamento de doenças (AMOROZO, 2002). Além disso, existe a falta de medicamento em locais de difícil acesso e a crença no potencial dos chamados produtos naturais, associada à propaganda "de que faz bem" (SILVA, 2003).

Estudos com plantas medicinais e seus usos foram realizados em diferentes regiões brasileiras (MACIEL et al., 2002; MACEDO; FERREIRA, 2004; TOSTI; COLLI, 2007; CRUZ-SILVA et al., 2009; GIRALDI; HANAZAKI, 2010; TULER, 2011; OLIVEIRA; MENINI, 2012; ALVES; POVH, 2013) e na região Nordeste, especificamente, podemos destacar alguns trabalhos, tais como os de Moreira et al. (2002), Morais et al. (2005), Pinto et al. (2006), Rodrigues e Guedes (2006), Teixeira e Melo (2006), Roque et al. (2010) e Almeida et al. (2012).

No Piauí, os estudos etnobotânicos publicados incluindo uso de plantas medicinais são relativamente recentes (SILVA, 2010) podendo-se registrar os trabalhos de Franco e Barros (2006), Chaves e Barros (2008; 2012), Oliveira (2008), Amorim (2010), Oliveira et al. (2010a), Sousa (2010), Aguiar e Barros (2012) e Baptistel et al. (2014). Estes estudos tornam-se importantes por revelar o uso destas plantas em um estado ainda tão desconhecido, tanto do ponto de vista florístico, quanto do conhecimento das espécies vegetais úteis ao homem para diferentes fins.

Dessa forma, almejando contribuir com o incremento das informações, ainda incipientes, presentes até o momento no panorama etnobotânico piauiense, este trabalho teve como objetivo realizar o levantamento das espécies vegetais utilizadas como recurso terapêutico na 
comunidade rural de Curral Velho, município de Luís Correia, norte do Piauí. Esta área encontra-se ainda em estudo de sua flora nativa (dados não publicados), possuindo elementos florísticos de caatinga, cerrado e restinga, sugerindo que a comunidade vegetal na região costeira do Piauí possui natureza transicional, a qual está sofrendo ação antrópica, o que incentiva o conhecimento do uso, por parte da população que a habita, das espécies vegetais, principalmente nativas, no que se refere às suas indicações terapêuticas, partes utilizadas, formas de preparo, modos de administração e modos de obtenção. Almeja-se também que informações como estas tragam subsídios para contribuir com a valorização de perspectivas locais de conservação da fitodiversidade presente no seu entorno.

\section{Material e Métodos}

\section{Área de estudo}

O presente estudo foi desenvolvido na comunidade de Curral Velho (2॰58'0.33'S e 41³5.7'6”O), município de Luís Correia, Piauí, localizado na zona rural, a 21,9 $\mathrm{km}$ da sede do município, com acesso na altura do $\mathrm{km}$ 13,7, pela PI 315. A comunidade é composta por 76 residências, as quais estão distribuídas distanciadas umas das outras. O povoado possui uma escola que atende a estudantes do local e das regiões vizinhas e possui um posto de saúde, bem como acompanhamento de um agente de saúde às residências. Os seus habitantes se caracterizam, de um modo geral, pela origem essencialmente rural. Apresentam uma base econômica concentrada na agricultura, representada pela produção de feijão, milho, mandioca, e, no ramo da pecuária, criação de gados bovinos, caprinos, suínos e aves. Desenvolvem artesanatos utilizando algumas espécies de planta, como por exemplo a taboa (Typha domingensis Pers.).

\section{Coleta e análise de dados}

Os dados etnobotânicos foram obtidos mediante entrevista com a aplicação de 38 formulários semiestruturados, seguindo o preconizado por Albuquerque e Lucena (2004). Para critérios de escolha levaram-se em consideração os moradores da região com idade superior a 18 anos e que residam no local a mais de 15 anos, tempo de residência esse favorável para vivenciar as transformações ocorridas e absorver conhecimentos da flora local. Adotou-se a técnica de amostragem e seleção de informantes denominada "bola-de-neve" (BAYLEY, 1982), onde foram indicados informantes-chave da comunidade que possuíam maior conhecimento da flora. Nessa técnica, a primeira indicação de informante é indicada por um mediador local e as posteriores indicações são dadas pelos informantes entrevistados.

Os que aceitaram participar do trabalho assinaram o Termo de Consentimento Livre e Esclarecido autorizando a realização da pesquisa. Todos os informantes foram entrevistados individualmente, como recomendado por Phillip e Gentry (1993a), para evitar que as respostas fossem influenciadas por outro informante.

A entrevista e a "turnê guiada" (BERNARD, 1988) aconteceram paralelamente, dependendo da disponibilidade do entrevistado. Contou-se com a ajuda de moradores que possuíam notório conhecimento sobre a área e as plantas locais (mateiros), indicados pelo agente de saúde da comunidade, os quais passaram a ser os informantes-chave desta pesquisa (BAYLEY, 1982).

A coleta e herborização do material botânico foi realizada de acordo com Silva et al. (1989). Aidentificação botânica dos espécimes foi feita por meio do estudo da sua morfologia, consultas à bibliografia especializada, comparações com indivíduos já identificados e incorporados em herbário (AMORIM, 2010). O material botânico encontra-se tombado no acervo do Herbário da Universidade Federal do Piauí-UFPI/CMRV. A lista florística foi ordenada alfabeticamente por família, seguindo a proposta do Angiosperm Phylogeny Group III (APG III, 2009). A informação referente ao hábito das espécies seguiu Font Quer (1977). As sinonímias botânicas foram atualizadas utilizando-se a base de dados disponível na lista de espécies da flora do Brasil, de 2012 (http://floradobrasil.jbrj.gov.br/2012/index).

As informações obtidas foram agrupadas segundo a classificação das doenças da Organização Mundial da Saúde (OMS, 2000) e calculado o Fator de Consenso dos Informantes (FCI) de acordo com Troter e Logan 
(1986), utilizando a fórmula $\mathrm{FCI}=$ nur - nt $/$ nur -1 , onde, nur é número de citações de uso em cada categoria e nt=número de espécies usadas. Este processo teve como finalidade identificar as categorias de doenças que apresentam maior importância relativa local.

Para cada uma das espécies citadas foi calculado o seu valor de uso (VU), conforme Rossato et al. (1999), a partir da fórmula $\mathrm{VU}=\sum \mathrm{U} / \mathrm{n}$, sendo: $\mathrm{U}=$ número de citações (ou usos) da etnoespécie por informante; $\mathrm{n}=$ número de informantes que citaram a etnoespécie ou espécie. O termo etnoespécie nesse estudo significa a espécie útil para os moradores locais, reconhecida por um nome popular (ROSSATO et al., 1999).

Para cada espécie de planta, calculou-se a Importância Relativa (IR), com base na proposta de Bennett e Prance (2000). O cálculo da importância relativa, sendo 2 o valor máximo obtido por uma espécie, foi feito de acordo com a fórmula: $\mathrm{IR}=\mathrm{NSC}+\mathrm{NP}$ onde, NSC refere-se ao número de sistemas corporais e NP corresponde ao número de propriedades. O NSC é calculado pela fórmula NSC $=\mathrm{NSCE} / \mathrm{NSCEV}$, sendo que NSCE refere-se ao número de sistemas corporais tratados por uma determinada espécie e NSCEV é o número total de sistemas corporais tratados pela espécie mais versátil. O NP é calculado pela fórmula $\mathrm{NP}=\mathrm{NPE} /$ NPEV, onde NPE é o número de propriedades atribuídas para uma determinada espécie e NPEV é o número total de propriedades atribuídas à espécie mais versátil.

\section{Resultados e Discussão}

As entrevistas permitiram identificar 62 taxa distribuídos em 57 gêneros e 38 famílias botânicas (Tabela 1), dos quais 59 (95\%) foram identificados em nível de espécie e 3 (5\%) em nível de gênero. As famílias botânicas que apresentaram o maior número de espécies citadas foram: Fabaceae (13,11\%), Lamiaceae $(8,19 \%)$, Euphorbiaceae (6,55\%), Anacardiaceae, Myrtaceae e Rutaceae (com 4,91\%). Juntamente com outras, essas famílias também estão entre as mais abundantes em levantamentos realizados em diferentes lugares do Brasil, como por Medeiros et al. (2004), na Reserva Rio das Pedras, Mangaratiba (RJ); Costa (2005), em Castelo do Piauí (PI); Pinto et al. (2006), em Itacaré (BA); Oliveira
(2008), em comunidades rurais do Semiárido Piauiense; Borges e Peixoto (2009), em uma comunidade caiçara do litoral sul do estado do Rio de Janeiro; Roque et al. (2010), na comunidade rural de Laginhas, município de Caicó (RN); Oliveira e Menini (2012), no povoado de Manejo; Lima Duarte (MG) e por Alves e Povh (2013) na comunidade de Santa Rita, Ituiutaba (MG).

Apesar de todas as partes das plantas terem sido mencionadas pelos entrevistados, as partes mais utilizadas foram as folhas $(50,36 \%)$, para todas as doenças citadas, seguido da casca $(28,46 \%)$, fruto e raiz (ambos com 7,05\%), flor (3,16\%) e semente $(2,91 \%)$. Resultados semelhantes referentes à maior utilização das folhas foram registrados por Franco e Barros (2006), no Quilombo de Olho D'água dos Pires, Esperantina (PI) e Alves e Povh (2013), na comunidade de Santa Rita, Ituiutaba (MG). Castellucci et al. (2000) observaram que o maior uso das folhas pode estar relacionado ao fato dessas serem mais fáceis para coletar, além de estarem presentes na maior parte do ano.

O modo de administrar mais comum é por via oral $(94,64 \%)$, tópico $(4,37 \%)$ e inalação $(0,73 \%)$. Durante as entrevistas, foram citadas três formas de preparar chás, que segundo Simões et al. (1989) podem ser classificados em infusão, decocção e maceração. O chá em forma de decocção foi o que teve maior indicação (27\%), seguido da maceração $(23,11 \%)$ e da infusão $(17,51 \%)$.

Além dos chás, ainda utilizam sucos (8\%), garrafadas $(6,56 \%)$, in natura $(2,91 \%)$, cataplasma $(2 \%)$ (aplicação da erva diretamente na parte dolorida, inchada ou ferida) e banho como menor utilização $(0,73 \%)$. Chás na forma de decocção também foram predominantes nos estudos de Moreira et al. (2002), Pinto et al. (2006) e Oliveira et al. (2010a). Apesar da técnica de decocção ser aplicada geralmente para o preparo de chás das cascas, raízes ou pedaços de caule, na comunidade Curral Velho é comum a utilização da decocção com folhas, pois os informantes acreditam ser um método eficaz para a extração dos compostos benéficos presentes na planta. Em estudos realizados por Medeiros et al. (2004) e Tuler (2011) na região Sudeste do Brasil, observa-se também essa mesma incidência na utilização das folhas e do decocto, na preparação dos medicamentos. 
TABELA 1: Espécies usadas medicinalmente pelos moradores da comunidade de Curral Velho, Luís Correia, Piauí. NVNome Vulgar; H-Hábito: erv-erva; sub-subarbusto; arb-arbusto, arv-árvore, trep-trepadeira; NC-número de coletor de JLA; VU-Valor de Uso; IR-Importância Relativa; il-identificada no local.

\begin{tabular}{|c|c|c|c|c|c|}
\hline Família/Espécie & NV & & $\mathrm{NC}$ & VU & IR \\
\hline \multicolumn{6}{|l|}{ Acanthaceae } \\
\hline Justicia pectoralis Jacq. & anador & erv & 52 & 1,00 & 1,00 \\
\hline \multicolumn{6}{|l|}{ Anacardiaceae } \\
\hline Anacardium occidentalis $\mathrm{L}$. & cajueiro & arv & 16 & 1,22 & 1,38 \\
\hline Mangifera indica $\mathrm{L}$. & mangueira & arv & 60 & 1,00 & 0,38 \\
\hline Myracrodruon urundeuva Allemão & aroeira & arv & 18 & 1,06 & 1,75 \\
\hline \multicolumn{6}{|l|}{ Annonaceae } \\
\hline Annona muricata $\mathrm{L}$. & araticum & arv & 58 & 2,00 & 0,50 \\
\hline Annona squamosa L. & ata & arv & 48 & 2,00 & 0,75 \\
\hline \multicolumn{6}{|l|}{ Arecaceae } \\
\hline $\begin{array}{l}\text { Copernicia prunifera (Mill.) H.E. Moore } \\
\text { Aristolochiaceae }\end{array}$ & carnaúba & arb & 49 & 1,50 & 1,13 \\
\hline $\begin{array}{l}\text { Aristolochia triangularis Cham. \& Schltdl } \\
\text { Asparagaceae }\end{array}$ & angelicó & trep & 42 & 3,00 & 0,50 \\
\hline $\begin{array}{l}\text { Asparagus sp. } \\
\text { Bignoniaceae }\end{array}$ & milindro & erv & 56 & 1,00 & 0,38 \\
\hline $\begin{array}{l}\text { Tabebuia impetiginosa (Mart. ex DC.) Standl. } \\
\text { Bixaceae }\end{array}$ & pau-d'arco & arv & 59 & 1,00 & 0,38 \\
\hline $\begin{array}{l}\text { Bixa orellana } \mathrm{L} . \\
\text { Caricaceae }\end{array}$ & urucum & arb & 44 & 2,00 & 0,38 \\
\hline $\begin{array}{l}\text { Carica papaya } \mathrm{L} . \\
\text { Chenopodiaceae }\end{array}$ & mamão & arv & 51 & 1,00 & 0,38 \\
\hline $\begin{array}{l}\text { Chenopodium ambrosioides L. } \\
\text { Combretaceae }\end{array}$ & mastruz & erv & 32 & 1,83 & 0,75 \\
\hline Combretum leprosun Mart. & mufumbo & arb & 02 & 1,20 & 1,50 \\
\hline Terminalia catappa $\mathrm{L}$. & amêndoa & $\operatorname{arv}$ & 11 & 1,00 & 0,50 \\
\hline \multicolumn{6}{|l|}{ Costaceae } \\
\hline \multicolumn{5}{|l|}{ Crassulaceae } & 0,38 \\
\hline Bryophyllum pinnatum (Lam.) Omen & corana & erv & 43 & 1,25 & 1,13 \\
\hline \multicolumn{6}{|l|}{ Cucurbitaceae } \\
\hline \multicolumn{5}{|l|}{ Euphorbiaceae } & 0,38 \\
\hline Croton blanchetianus Baill. & mameleiro & arb & 01 & 1,25 & 1,50 \\
\hline Jatropha curcas L. & pião manso & arb & 31 & 1,00 & 0,38 \\
\hline Jatropha molissima (Pohl) Baill. var.mollissima & pião bravo & arb & 45 & 1,00 & 0,38 \\
\hline Phyllanthus niruri L. & quebra pedra & erv & 10 & 1,00 & 0,50 \\
\hline \multicolumn{6}{|l|}{ Fabaceae (Caesalpinioideae) } \\
\hline Bauhinia cheilantha (Bong.) Steud. & mororó & arb & 03 & 1,20 & 1,13 \\
\hline Copaifera langsdorfii Desf. & podoi & arv & 04 & 1,50 & 0,88 \\
\hline Hymenaea courbaril $\mathrm{L}$. & jatobá & arv & 19 & 1,38 & 1,25 \\
\hline Libidibia ferrea (Mart. ex Tul.) L.P.Queiroz. & jucá & arv & 17 & 1,10 & 0,88 \\
\hline Poincianella pyramidalis (Tul) L.Queiroz & catingueira & arv & 39 & 1,11 & 1,75 \\
\hline \multicolumn{6}{|l|}{ Fabaceae (Mimosoideae) } \\
\hline Tamarindus indica $\mathrm{L}$. & tamarina & arv & 13 & 2,00 & 0,75 \\
\hline Acacia farnesiana Wall. & coronha & arb & 43 & 1,25 & 0,38 \\
\hline Mimosa caesalpiniifolia Benth. & sabiá & arb & 15 & 1,50 & 0,75 \\
\hline \multicolumn{6}{|l|}{ Iridaceae } \\
\hline Eleutherine bulbosa (Mill.)Urb. & palmeirinha & erv & 14 & 1,50 & 0,38 \\
\hline
\end{tabular}




\begin{tabular}{|c|c|c|c|c|c|}
\hline \multicolumn{6}{|l|}{ Lamiaceae } \\
\hline Mentha $x$ villosa Huds & hortelã & erv & 37 & 1,35 & 1,63 \\
\hline Ocimum gratissimum $\mathrm{L}$. & alfavaca & sub & 23 & 2,00 & 0,75 \\
\hline Plectranthus barbatus Andrew. & boldo & erv & 20 & 1,05 & 1,75 \\
\hline Ocimum brasilicum L. & manjericão & sub & 22 & 1,00 & 0,38 \\
\hline Plectranthus amboinicus (Lour.) Spreng. & malva & erv & 21 & 1,36 & 1,38 \\
\hline \multicolumn{6}{|l|}{ Liliaceae } \\
\hline \multicolumn{6}{|l|}{ Loranthaceae } \\
\hline Struthanthus sp. & enxerco & erv & 06 & 1,00 & 0,38 \\
\hline \multicolumn{4}{|l|}{ Malvaceae } & 1,00 & 0,38 \\
\hline \multicolumn{4}{|l|}{ Moringaceae } & 1,75 & 1,75 \\
\hline \multicolumn{4}{|l|}{ Musaceae } & 1,50 & 0,88 \\
\hline \multicolumn{4}{|l|}{ Myrtaceae } & 1,00 & 0,50 \\
\hline Psidium guajava $\mathrm{L}$. & goiaba & arv & 12 & 1,00 & 0,38 \\
\hline Syzygium cumini (L.) Skeels & azeitona & arv & 46 & 1,00 & 0,75 \\
\hline \multicolumn{5}{|l|}{ Nyctaginaceae } & 0,75 \\
\hline $\begin{array}{l}\text { Boerhavia diffusa } \mathrm{L} \text {. } \\
\text { Olacaceae }\end{array}$ & pega pinto & erv & 09 & 1,00 & 0,75 \\
\hline \multicolumn{5}{|l|}{ Oxalidaceae } & 1,75 \\
\hline Averrhoa carambola $\mathrm{L}$. & carambola & arv & 61 & 1,00 & 0,75 \\
\hline \multicolumn{5}{|l|}{ Phytolaccaceae } & 0,38 \\
\hline \multicolumn{5}{|l|}{ Piperaceae } & 1,13 \\
\hline \multicolumn{5}{|l|}{ Plantaginaceae } & 0,38 \\
\hline \multicolumn{5}{|l|}{ Poaceae } & 1,13 \\
\hline $\begin{array}{l}\text { Cymbopogon citratus (DC.) Stapf. } \\
\text { Punicaceae }\end{array}$ & capim limão & erv & 34 & 1,00 & 1,75 \\
\hline \multicolumn{5}{|l|}{ Rubiaceae } & 0,63 \\
\hline \multicolumn{6}{|l|}{ Rutaceae } \\
\hline Citrus $x$ aurantium $\mathrm{L}$. & laranja & arv & 53 & 1,25 & 1,38 \\
\hline Citrus limonum Risso. & limão & arv & 26 & 1,00 & 0,38 \\
\hline \multicolumn{6}{|l|}{ Turneraceae } \\
\hline & \multicolumn{4}{|c|}{ Verbenaceae } & 2,00 \\
\hline Stachytarpheta cayennensis (Rich.) Vahl & cataflam & erv & 55 & 3,00 & 0,88 \\
\hline $\begin{array}{l}\text { Lippia alba (Mill.) N.E.Br. } \\
\text { Zingiberaceae }\end{array}$ & erva cidreira & erv & 24 & 1,24 & 1,63 \\
\hline Alpinia nutans Rosc & jardineira & sub & 27 & 1,00 & 0,38 \\
\hline
\end{tabular}


As espécies que obtiveram maior número de citações foram Lippia alba (21), Plectranthus amboinicus e Plectranthus barbatus (19). Resultados semelhantes para $L$. alba e P. barbatus foi verificado por Castro et al. (2011). Essas três espécies também foram as mais citadas no estudo realizado por Cruz-Silva et al. (2009), no município de Quedas do Iguaçu (PR). Lippia alba é uma espécie citada pelos moradores locais como de grande utilidade como calmante, contra dor de cabeça e febre.

As plantas referidas encontram-se distribuídas em diferentes hábitos de crescimento, tais como herbáceo $(36,66 \%)$, arbustivo $(31,66 \%)$, arbóreo $(26,66 \%)$ e subarbustivo $(5,00 \%)$. Resultados semelhantes têm sido relatados em outras pesquisas (MOREIRA et al., 2002; PINTO et al., 2006; ALBERTASSE et al., 2010; BRATTI et al., 2013; LIPORACCI; SIMÃO, 2013). Pilla et al. (2006) relatam que a predominância de plantas com hábito herbáceo na medicina popular pode estar relacionada ao fato delas serem cultivadas geralmente nos quintais, o que facilita a obtenção desses recursos vegetais. De fato, as plantas medicinais conhecidas na comunidade de Curral Velho são obtidas, principalmente, nos quintais ou em áreas próximas. O costume de cultivar principalmente ervas nos quintais facilita o uso destas plantas. Por outro lado, Meireles (2012), em estudo semelhante na comunidade Canárias, Araioses (MA), encontraram resultados diferentes deste, onde obteve maior representação de plantas com hábito arbóreo. A comunidade estudada possui em seu entorno uma área vegetal conservada, a qual possui espécies pertencentes às vegetações de caatinga, restinga e cerrado, o que explica a grande quantidade de árvores e arbustos nativos utilizados.

As espécies que apresentaram maior valor de uso foram Aristolochia triangularis, Petiveria alliaceae e Stachytarpheta cayennensis (VU = 3,00) (Tabela 1), as quais apresentam três usos medicinais distintos informados por um membro da comunidade.

Conforme pode ser observado na Tabela 1, as espécies de plantas que apresentaram grande versatilidade quanto a seus usos, verificados através do cálculo de Importância Relativa, foram Turnera subulata (IR 2,00), Gossypium hirsutum, Myracrodruon urundeuva, Ximenia americana, Plectranthus barbatus, Cymbopogon citratus, Poincianella pyramidalis e Chenopodium ambrosioides (todas com IR 1,75). T. subulata é uma das espécies que apresenta o maior número de usos terapêuticos, citada para três sistemas corporais, dentre os dez sistemas corporais registrados na área de estudo.

Segundo Friedman et al. (1986), uma planta com índice de importância relativamente alto pode sugerir uma real efetividade no tratamento da doença, pois em estudos etnobotânicos esse índice facilitará a seleção de espécies para testes farmacológicos que possam vir a provar uma eficácia de seus princípios ativos.

Os sintomas e doenças foram enquadrados em dez categorias classificadas segundo a Organização Mundial da Saúde. As categorias que apresentaram maior importância relativa, levando em consideração o índice Fator de Consenso dos Informantes (FCI), foram S00-T98 ( $\mathrm{FCI}=0,80), \mathrm{R} 50-\mathrm{R} 69$, (FCI=0,72), J00-J99 (FCI=0,70), K00-K93 (FCI=0,66), I00-I99 (FCI=0,93) (Tabela 2). A categoria que apresentou menor índice foi C00-D48 $(\mathrm{FCI}=0)$. Isso acontece por essa categoria não estar relacionada a enfermidades corriqueiras para a maioria dos informantes. Esses resultados se assemelham ao estudo de Meireles (2012), desenvolvido na comunidade Canárias, Araioses (MA), onde, das oito categorias mais representativas, três também foram registradas na comunidade de Curral Velho. Doenças relacionadas com os aparelhos circulatório, respiratório e digestivo também apresentam o índice FCI relevante no estudo feito por Santos (2010). No trabalho feito por Brito e SennaValle (2011) na comunidade caiçara da Praia do Sono, Paraty (RJ), as categorias de doenças que tiveram maior importância relativa foram as relacionadas a doenças do aparelho digestivo e respiratório. Das 13 categorias de doenças encontradas no trabalho de Amorozo (2002), nove estão presentes nesse trabalho, sendo as mais representativas as relacionadas a doenças do aparelho digestivo e respiratório. Diferentemente dos resultados desse estudo, Silva (2010), em seu trabalho desenvolvido em comunidades rurais da Serra de Campo Maior (PI), obteve maior FCI para doenças do fígado, transtorno neurótico e do aparelho geniturinário. Os resultados de Oliveira et al. (2010b), na comunidade urbana de Muribeca (PE), também diferiram dos obtidos nessa pesquisa onde as categorias com maior fator de consenso foram as relacionadas a doenças parasitárias, sistemas nervoso e gastrintestinal. 
Dos sistemas corporais tratados com as plantas medicinais, o que obteve maior número de espécies na área estudada foi R50-R69, com 28,86\%; J00-J99, com 22,15\%, N00-N99, com 13,42\% e K00-K93 com 12,75\% (Tabela 2). Resultados semelhantes têm sido evidenciados em outros estudos (ALMEIDA; ALBUQUERQUE, 2002; GIRALDI; HANAZAKI, 2010), os quais se referem às categorias mais representativas relacionadas a doenças e sintomas dos sistemas digestório, respiratório e geniturinário.

Em suma, verificou-se que a comunidade estudada possui um importante repertório sobre plantas medicinais que tem sido fundamental no tratamento de doenças, que, conjuntamente aos dados já existentes para o estado, poderão fornecer um panorama mais geral para a tomada de decisões mais contundentes necessárias à conservação da flora local.

Além de demonstrar a riqueza de conhecimento sobre plantas medicinais, este estudo indica também que, em um estado do nordeste brasileiro onde os recursos financeiros para tratamento de doenças e o funcionamento do sistema público de saúde são bastante precários, torna-se fundamental estudar o potencial medicinal dos recursos vegetais do estado.

TABELA 2: Fator de Consenso dos Informantes (FCI) sobre as plantas medicinais na comunidade de Curral Velho, Piauí, Brasil, seguindo a classificação da Organização Mundial da Saúde a qual se baseia em sistemas corporais.

\begin{tabular}{|c|c|c|}
\hline Código/FCI/Sistema & Espécies medicinais & Indicação \\
\hline $\begin{array}{l}(\mathrm{S} 00-\mathrm{T} 98) \mathrm{FCI}=0,80 \\
\text { Lesões, envenenamento e algumas outras } \\
\text { consequências de causas externas }\end{array}$ & $\begin{array}{l}\text { Chenopodium ambrosioides } \\
\text { Genipa americana } \\
\text { Petiveria alliaceae } \\
\text { Libidibia ferrea }\end{array}$ & $\begin{array}{l}\text { fratura/pancadas } \\
\text { luxação } \\
\text { pancadas } \\
\text { pancadas }\end{array}$ \\
\hline $\begin{array}{l}(\mathrm{R50}-\mathrm{R69}) \mathrm{FCI}=0,72 \\
\text { Sintomas e sinais gerais }\end{array}$ & $\begin{array}{l}\text { Cymbopogon citratus } \\
\text { Lippia alba } \\
\text { Mentha x villosa } \\
\text { Citrus aurantium } \\
\text { Myracrodruon urundeuva } \\
\text { Anacardium occidentalis } \\
\text { Hymenaea stigonocarpa } \\
\text { Libidibia ferrea } \\
\text { Croton blanchetianus } \\
\text { Chenopodium ambrosioides } \\
\text { Combretum leprosum } \\
\text { Jatropha mollissima } \\
\text { Ruta graveolens } \\
\text { Oxalis regnellii } \\
\text { Citrullus sp. } \\
\text { Justicia pectoralis } \\
\text { Aristolochia triangularis } \\
\text { Plectranthus barbatus } \\
\text { Eleutherine bulbosa } \\
\text { Ocimum gratissimum } \\
\text { Stachytarpheta cayennensis } \\
\text { Jatropha curcas } \\
\text { Gossypium hirsutum } \\
\text { Acacia farnesiana } \\
\text { Eucalyptus globulus } \\
\text { Annona squamosa } \\
\text { Turnera subulata } \\
\text { Ximenia americana } \\
\text { Annona muricata } \\
\text { Aloe vera } \\
\text { Bryophyllum pinnatum } \\
\text { Bauhinia cheilantha } \\
\text { Scoparia dulcis } \\
\text { Copernicia prunifera } \\
\text { Petiveria alliaceae } \\
\text { Poincianella pyramidalis } \\
\text { Copaifera langsdorfii } \\
\text { Mimosa caesalpiniifolia }\end{array}$ & $\begin{array}{l}\text { calmante/dor de barriga } \\
\text { calmante } \\
\text { calmante/cólica infantil/dor de barriga/febre } \\
\text { calmante/ ensoo } \\
\text { cicatrização/infecção/inflamação } \\
\text { cicatrização/infecção/inflamação } \\
\text { cicatrização/infecção/inflamação } \\
\text { cicatrização/inflamação } \\
\text { cicatrização } \\
\text { cicatrização/inflamação/dor nas costas } \\
\text { cicatrização } \\
\text { cicatrização } \\
\text { dor de barriga/dor de cabeça } \\
\text { cólica } \\
\text { convulsão } \\
\text { dor/dor de cabeça/febre } \\
\text { dor de barriga/dor nos ossos } \\
\text { dor de barriga/ressaca } \\
\text { dor de barriga } \\
\text { dor de barriga } \\
\text { dor de cabeça/dor na coluna } \\
\text { dor de cabeça } \\
\text { dor de ouvido/inflamação } \\
\text { febre } \\
\text { febre } \\
\text { indutor de vômito } \\
\text { inflamação/infecção } \\
\text { inflamação } \\
\text { inflamação/insônia } \\
\text { inflamação } \\
\text { inflamação } \\
\text { inflamação } \\
\text { inflamação } \\
\text { resguardo quebrado } \\
\text { reumatismo } \\
\text { reumatismo } \\
\text { reumatismo } \\
\text { reumatismo }\end{array}$ \\
\hline
\end{tabular}


(J00-J99) FCI $=0,70$

Doenças do aparelho respiratório

(K00-K93) FCI =0,66

Doenças do aparelho digestivo
Musa paradisiaca

Hymenaea stigonocarpa

Croton blanchetianus

Plectranthus amboinicus

Punica granatum

Malpighia glabra

Ocimum gratissimum

Gossypium hirsutum

Ximenia americana

Justicia pectoralis

Poincianella pyramidalis

Bryophyllum pinnatum

Lippia alba

Eucalyptus globulus

Mentha x villosa

Struthanthus sp.

Citrus aurantium

Citrus limon

Bixa orellana

Combretum leprosum

Carica papaya

Bauhinia cheilantha

Stachytarpheta cayennensis

Ocimum brasilicum

Mimosa caesalpiniifolia

Anacardium occidentalis

Psidium guajava

Croton blanchetianus

Poincianella pyramidalis

Cymbopogon citratus

Plectranthus barbatus

Ximenia americana

Myracrodruon urundeuva

Piper tuberculatum

Citrus aurantium

Combretum leprosun

Copaifera langsdorfi

Tamarindus indica

Annona squamosa

Scoparia dulcis

Asparagus sp.

Poincianella pyramidalis

Bauhinia cheilantha

Tamarindus indica

Cymbopogon citratus

Lippia alba

Ruta graveolens

Petiveria alliaceae

Gossypium hirsutum

Plectranthus amboinicus

Costus spicatus

Averrhoa carambola

Phyllanthus niruri

Terminalia catappa

Turnera subulata

Boerhavia diffusa

Syzygium cumini

Bixa orellana

Ximenia americana

Aristolochia triangularis

Bryophyllum pinnatum asma/tosse

bronquite/gripe/pneumonia

gripe

gripe/dor de garganta

inflamação de garganta

gripe

gripe

gripe

gripe

gripe

gripe/tosse /tuberculose

gripe

gripe

gripe

gripe

gripe

gripe

gripe

gripe

gripe

gripe

gripe

gripe/inflamação de garganta

sinusite

tosse

inflamação na garganta

diarreia

diarreia/gastrite/intestino/má digestão

estômago

estômago

estômago/má digestão/fígado

gases

gases

gases

gastrite/intestino

gastrite/estômago

gastrite/má digestão

laxante

má digestão

má digestão

coração

derrame

hemorroidas

hemorroidas

pressão alta

pressão alta

cólica menstrual

cólica menstrual

corrimento/ inflamação no útero e nos ovários

corrimento/inflamação no útero e nos ovários

dor nos rins/inflamação nos rins

dor nos rins/inflamação nos rins

pedra nos rins

inflamação nos rins

inflamação nos rins/inflamação no útero

infecção urinária

infecção urinária

inflamação na próstata

menstruação irregular

menstruação irregular

inflamação no útero 


\begin{tabular}{|c|c|c|}
\hline $\begin{array}{l}\text { (L00-L99) FCI }=0,25 \\
\text { Doenças de pele e do tecido subcutâneo: } \\
\text { dermatite, unhas }\end{array}$ & $\begin{array}{l}\text { Cymbopogon citratus } \\
\text { Turnera subulata } \\
\text { Alpinia nutans }\end{array}$ & $\begin{array}{l}\text { coceira } \\
\text { coceira/furúnculo } \\
\text { coceira }\end{array}$ \\
\hline $\begin{array}{l}\text { (E00-E90) FCI }=0,20 \\
\text { Doenças endócrinas, nutricionais, } \\
\text { metabólicas }\end{array}$ & $\begin{array}{l}\text { Syzygium cumini } \\
\text { Moringa citrifolia } \\
\text { Anacardium occidentalis }\end{array}$ & $\begin{array}{l}\text { colesterol } \\
\text { colesterol/diabetes } \\
\text { diabetes }\end{array}$ \\
\hline $\begin{array}{l}\text { (D50-D89) FCI=0,13 } \\
\text { Doenças do sangue e dos órgãos } \\
\text { hematopoéticos e alguns transtornos } \\
\text { imunitários }\end{array}$ & $\begin{array}{l}\text { Myracrodruon urundeuva } \\
\text { Copernicia prunifera } \\
\text { Boerhavia diffusa } \\
\text { Averrhoa carambola } \\
\text { Mangifera indica }\end{array}$ & $\begin{array}{l}\text { afinar o sangue } \\
\text { afinar o sangue } \\
\text { afinar o sangue } \\
\text { anemia } \\
\text { hepatite }\end{array}$ \\
\hline $\begin{array}{l}(\mathrm{C} 00-\mathrm{D} 48) \mathrm{FCI}=0 \\
\text { Neoplasias (tumores) }\end{array}$ & $\begin{array}{l}\text { Gossypium hirsutum } \\
\text { Aloe vera } \\
\text { Bixa orellana } \\
\text { Moringa citrifolia }\end{array}$ & $\begin{array}{l}\text { câncer } \\
\text { câncer } \\
\text { câncer } \\
\text { câncer }\end{array}$ \\
\hline
\end{tabular}

\section{Agradecimentos}

Os autores agradecem à comunidade de Curral Velho pela receptividade e interesse demonstrados, à Dra. Rosemary Sousa pela leitura crítica realizada neste artigo e aos avaliadores anônimos pelas valiosas sugestões emitidas.

\section{Referências}

AGUIAR, L. C. G. G.; BARROS, R. F. M. Plantas medicinais cultivadas em quintais de comunidades rurais no domínio do cerrado piauiense (município de Demerval Lobão, Piauí, Brasil). Revista Brasileira de Plantas Medicinais, Botucatu, v. 14, n. 3, p. 419-434, 2012.

ALBERTASSE, P. D.; THOMAZ, L. D.; ANDRADE, M. A. Plantas medicinais e seus usos na comunidade da Barra do Jucu, Vila Velha, ES. Revista Brasileira de Plantas Medicinais, Botucatu, v. 12, n. 3, p. 250-260, 2010.

ALBUQUERQUE, U. P. Introdução a Etnobotânica. 2005. Disponível em: <http://www.scielo.br/scielo.php?script=sci nlink s\&ref $=000084 \&$ pid $=\mathrm{S} 15160572201300040000900003 \& \operatorname{lng}=$ en $>$. Acesso em: 16 maio 2014.

ALBUQUERQUE, U. P.; LUCENA, R. F. P. Métodos e técnicas na pesquisa etnobotânica. Recife: Livro Rápido/NUPEEA, 2004. $189 \mathrm{p}$.

ALMEIDA, C. de F. C. B. R.; ALBUQUERQUE, U. P. Uso e conservação de plantas e animais medicinais no estado de Pernambuco (Nordeste do Brasil): um estudo de caso. Interciência, Caracas, v. 27, n. 6, p. 276-285, 2002.

ALMEIDA, C. de F. C. B. R.; RAMOS, M. A.; SILVA, R. R. V.; MELO, J. G. de; MEDEIROS, M. F. T.; ARAÚJO, T. A. de S.; ALMEIDA, A. L. S. de; AMORIM, E. L. C. de; ALVES, R. R. da N.; ALBUQUERQUE, U. P. de. Intracultural variation in the knowledge of medicinal plants in a urban-rural community in the Atlantic Forest from Northeastern Brazil. Evidence-Based Complementary and Alternative Medicine, New York, v. 2012, p. 1-15, 2012.
ALVES, G. S. P; POVH, J. P. Estudo etnobotânico de plantas medicinais na comunidade de Santa Rita, Ituiutaba - MG. Biotemas, Florianópolis, v. 26, n. 3, p. 231-242, 2013.

AMORIM, A. N. Etnobiologia da comunidade de pescadores artesanais urbanos do bairro Poti Velho, Teresina/PI, Brasil. 2010. 122 f. Dissertação (Mestrado em Desenvolvimento e Meio Ambiente) - Universidade Federal do Piauí, Teresina. 2010.

AMOROZO, M. C. M. Uso e diversidade de plantas medicinais em Santo Antônio do Laverger, MT, Brasil. Acta Botanica Brasilica, Feira de Santana, v. 16, n. 2, p. 189-203, 2002.

APG III - ANGIOSPERM PHYLOGENY GROUP III. An update of the Angiosperm phylogeny group classification for the orders and families of flowering plants: APG III. Botanical Journal of the Linnean Society, London, v. 161, p. 105-121, 2009.

BADKE, M. R.; BUDÓ, M. L. D.; SILVA, F. M.; RESSEL, L. B. Plantas medicinais: o saber sustentado na prática do cotidiano popular. 2011. Disponível em: <http://www.scielo.br/pdf/ean/ v15n1/19.pdf.> Acesso em: 6 jun. 2014.

BAPTISTEL, A. C.; COUTINHO, J. M. C. P.; LINS NETO, E. M. F.; MONTEIRO, J. M. Plantas medicinais utilizadas na comunidade Santo Antônio, Currais, Sul do Piauí: um enfoque etnobotânico. Revista Brasileira de Plantas Medicinais, Campinas, v. 16, n. 2, supl. I, p. 406-425, 2014.

BAYLEY, K. D. Methods of social research. New York: Free Press, 1982. 553 p.

BENNETT, B. C.; PRANCE, G. T. Introduced plants in the indigenous pharmacopoeia of Northern South America. Economic Botany, New York, v. 54, n. 1, p. 90-102, 2000.

BERNARD, H. R. Research methods in cultural anthropology. Newbury Park: Sage Publ, 1988. 520 p.

BORBA, A. M.; MACEDO, M. Plantas medicinais usadas para a saúde bucal pela comunidade do bairro Santa Cruz, Chapada dos Guimarães, MT, Brasil. Acta Botanica Brasilica, Feira de Santana, v. 20, n. 4, p. 771-782, 2006.

BORGES, R.; PEIXOTO, A. L. Conhecimento e uso de plantas em uma comunidade caiçara do litoral sul do estado do Rio de Janeiro, Brasil. Acta Botanica Brasilica, Feira de Santana, v. 23, n. 3, p. 769-779, 2009. 
BRATTI, C.; VIEIRA, M. C.; ZÁRATE, N. A. H.; OLIVEIRA, A. P. A.; MARAFIGA, B. G.; FERNANDES, S. S. L. Inventory of native medicinal plants on a farm in Dourados-MS. Revista Brasileira de Plantas Medicinais, Botucatu, v. 15, n. 4, p. 675-683, 2013.

BRITO, M. R.; SENNA-VALLE, L. Plantas medicinais utilizadas na comunidade caiçara da Praia do Sono, Paraty, Rio de Janeiro, Brasil. Acta Botanica Brasilica, Feira de Santana, v. 25, n. 2, p. 363-372. 2011.

CASTELLUCCI, S.; LIMA, M. I. S.; NORDI, N.; MARQUES, J. G. W. Plantas medicinais relatadas pela comunidade residente na Estação Ecológica de Jataí, município de Luís Antonio-SP; uma abordagem etnobotânica. Revista Brasileira de Plantas Medicinais, Botucatu, v. 3, n. 1, p. 51-60, 2000.

CASTRO, C. K. C.; CRUZ, L. T. C.; PASA, M. C. Uma abordagem etnobotânica dos conhecimentos populares com moradores da Serra 3 em Cuiabá, MT, Brasil. Revista Biodiversidade, Rondonópolis, v. 10 , n. 1, p. 80-90, 2011.

CHAVES, E. M. F.; BARROS, R. F. M. Resource use of the flora of the brushwood vegetation in Cocal in county. Piauí, Brasil. Functional Ecosystems and Communities, Middlesex, v. 2, Special Issue, p. 51-58, 2008.

CHAVES, E. M. F.; BARROS, R. F. M. Diversidade e uso de recursos medicinais do carrasco na APA da Serra da Ibiapaba, Piauí, Nordeste do Brasil. Revista Brasileira de Plantas Medicinais, Botucatu, v. 14, n. 2, p. 476-486, 2012.

COSTA, J. M. Estudo fitossociológico e sócio-ambiental de uma área de cerrado com potencial melitófilo no município de Castelo do Piauí, Piauí, Brasil. 2005. 109 f. Dissertação (Mestrado em Desenvolvimento e Meio Ambiente) - Universidade Federal do Piauí, Teresina. 2005.

COSTA, V. P.; MAYWORM, M. A. S. Plantas medicinais utilizadas pela comunidade do bairro dos Tenentes - município de Extrema, MG, Brasil. Revista Brasileira de Plantas Medicinais, Botucatu, n. 3, v. 13, p. 282-292, 2011.

CRUZ-SIlVA, C. T. A; PELINSON, A. P.; CAMPELO, A. M. Abordagem Etnobotânica acerca do uso de plantas medicinais na região urbana no município de Quedas do Iguaçu, Paraná. Cultivando o Saber, Cascavel, v. 2, n. 1, p. 14-25, 2009.

FONT QUER, M. P. Diccionario de Botánica. Barcelona: Labor, 1977. 1244 p.

FRANCO, E. A. P.; BARROS, R. F. M. Uso e diversidade de plantas medicinais no Quilombo Olho D'água dos Pires, Esperantina, Piauí. Revista Brasileira de Plantas Medicinais, Botucatu, v. 8, n. 3, p. 78-88, 2006.

FRIEDMAN, J.; YANIV, Z.; DAFNI, A.; PALEWITCH, D. A. A preliminary classification or the healing potential of medicinal plants based on a rational analysis of an etnopharmacological field survey among Beduins in the Negev desert, Israel. Journal of Ethnopharmacology, Shannon, v. 16, p. 275-287, 1986.

GIRALDI, M.; HANAZAKI, N. Uso e conhecimento tradicional de plantas medicinais no Sertão do Ribeirão, Florianópolis, SC, Brasil. Acta Botanica Brasilica, Feira de Santana, v. 24, n. 2, p. 395-406, 2010.

LIPORACCI, H. S. N.; SIMÃO, D. G. Levantamento etnobotânico de plantas medicinais nos quintais do Bairro Novo Horizonte, Ituiutaba, Revista Brasileira de Plantas Medicinais, Botucatu, v. 15, n. 4, p. 529-540, 2013.
LORENZI, H.; MATOS, F. J. A. Plantas medicinais no Brasil: nativas e exóticas. Nova Odessa: Instituto Plantarum, 2008. 544 p.

MACEDO, M.; FERREIRA, A. R. Plantas medicinais usadas para tratamentos dermatológicos, em comunidades da Bacia do Alto Paraguai, Mato Grosso. Revista Brasileira de Farmacognosia, Curitiba, v. 14, n. 1, p. 40-44, 2004.

MACIEL, M. A. M.; PINTO, A. C.; VEIGA JR., V. F. Plantas medicinais: a necessidade de estudos multidisciplinares. Química Nova, São Paulo, v. 25, n. 3, p. 429-438, 2002.

MEDEIROS, M. F. T.; FONSECA, V. S.; ANDREATA, R. H. P. Plantas medicinais e seus usos pelos sitiantes da Reserva Rio das Pedras, Mangaratiba, RJ, Brasil. Acta Botanica Brasilica, Feira de Santana, v. 18, n. 2, p. 391-399, 2004.

MEIRELES, V. J. S. Etnobotânica e caracterização da pesca na comunidade Canárias, Reserva Extrativista Marinha do Delta do Parnaíba, Nordeste do Brasil. 2012. 164 f. Dissertação (Mestrado em Desenvolvimento e Meio Ambiente) - Universidade Federal do Piauí, Teresina. 2012.

MORAIS, S. M.; DANTAS, J. D. P.; SILVA, A. R. A.; MAGALHÃES, E. F. Plantas medicinais usadas pelos índios Tapebas do Ceará. Revista Brasileira de Farmacognosia, Curitiba, v. 15, n. 2, p. 169-177, 2005.

MOREIRA, R. C. T.; COSTA, L. C. B.; COSTA, R. C. S.; ROCHA, E. A. Abordagem etnobotânica acerca do uso de plantas medicinais na Vila Cachoeira, Ilhéus, Bahia, Brasil. Acta Farmacêutica Bonaerense, Buenos Aires, v. 21, n. 3, p. 205-211, 2002.

NEGRELLE, R. R. B.; FORNAZZARI, K. R. C. Estudo etnobotânico em duas comunidades rurais (Limeira e Ribeirão Grande) de Guaratuba (Paraná, Brasil). Revista Brasileira de Plantas Medicinais, Botucatu, v. 9, n. 2, p. 36-54, 2007.

OLIVEIRA, E. R.; MENINI, N. L. Levantamento etnobotânico de plantas medicinais utilizadas pelos moradores do povoado de Manejo, Lima Duarte-MG. Revista Brasileira de Plantas Medicinais, Botucatu, v. 14, n. 2, p. 311-320, 2012.

OLIVEIRA, F. C. S. Conhecimento botânico tradicional em comunidades rurais do semiárido Piauiense. 2008. 134 f. Dissertação (Mestrado em Desenvolvimento e Meio Ambiente) Universidade Federal do Piauí, Teresina. 2008.

OLIVEIRA, F. C. S.; BARROS, R. F. M.; MOITA NETO, J. M. Plantas medicinais utilizadas em comunidades rurais de Oeiras, semiárido piauiense. Revista Brasileira de Plantas Medicinais, Botucatu, v. 12, n. 3, p. 282-301, 2010a.

OLIVEIRA, G. L.; OLIVEIRA, A. F. M.; ANDRADE, L. H. C. Plantas medicinais utilizadas na comunidade urbana de Muribeca, Nordeste do Brasil. Acta Botanica Brasilica, Feira de Santana, v. 24, n. 2, p. 571-577, 2010 b.

OMS - ORGANIZAÇÃO MUNDIAL DE SAÚDE. Classificação estatística internacional de doenças e problemas relacionados à saúde. 10. ed. São Paulo: Editora da Universidade de São Paulo, 2000. $1191 \mathrm{p}$.

PHILLIPS, O.; GENTRY, A. M. The useful plants of Tambopata, Peru. I. Statistical hypothesis with a new quantitative technique. Economic Botany, New York, v. 47, n. 1, p. 15-32, 1993a.

PHILLIPHS, O.; GENTRY, A. H.; REYNEL, C.; WILKIN, P.; GÁLVES-DURAND, B. C. Quantitative ethnobotany and amazonian conservation. Conservation Biology, San Francisco, v. 8, n. 1, p. 225-248, 1994. 
PILLA, M. A. C.; AMOROZO, M. C.; FURLAN, A. Obtenção e uso das plantas medicinais no distrito de Martim Francisco, município de Mogi-Mirim, SP, Brasil. Acta Botanica Brasilica, Feira de Santana, v. 20, n. 4, p. 789-802, 2006.

PINTO, E. P. P.; AMAROSO, M. C. M.; FURLAN, A. Conhecimento popular sobre plantas medicinais em comunidades rurais de mata atlântica - Itacaré, BA, Brasil. Acta Botanica Brasilica, Feira de Santana, v. 20, n. 4, p. 751-762, 2006.

RODRIGUES, A. C. C.; GUEDES, M. L. S. Utilização de plantas medicinais no Povoado Sapucaia, Cruz das Almas - Bahia. Revista Brasileira de Plantas Medicinais, Botucatu, v. 8, n. 2, p. 1-7, 2006.

RODRIGUES, J. S. C. Estudo etnobotânico das plantas aromáticas e medicinais. 2007. Disponível em: $<$ http://cbv.fc.ul. pt/PAM/pdfsLivro/JoanaCRodrigues.pdf. $>$. Acesso em: 13 mar 2013.

RODRIGUES, V. G. S. Cultivo, uso e manipulação de plantas medicinais. 2004. Disponível em: <http://www.infoteca.cnptia. embrapa.br/bitstream/doc/916031/1/doc91plantasmedicinais.pdf> Acesso em: 22 maio 2004.

ROQUE, A. A.; ROCHA, R. M.; LOIOLA, M. I. B. Uso e diversidade de plantas medicinais da Caatinga na comunidade rural de Lagoinhas, município de Caicó, Rio Grande do Norte (Nordeste do Brasil). Revista Brasileira de Plantas Medicinais, Botucatu, v. 12, n. 1, p. 31-42, 2010.

ROSSATO, S. C.; LEITÃO-FILHO, H. F.; BEGOSSI, A. Ethnobotany of caiçaras of the Atlantic Forest coast (Brazil). Economic Botany, New York, v. 53, n. 4, p. 387-395, 1999.

SANTOS, A. B. N. Uso e diversidade de plantas medicinais no munícipio de Cajueiro da Praia, Piauí, Brasil. 2010. $27 \mathrm{f}$. Monografia (Graduação em Ciências Biológicas) - Universidade Federal do Piauí, Parnaíba, 2010.

SILVA, A. T.; MUNIZ, C. F S.; WANDERLEY, M. G. L.; KIRIZAWA, M.; SENDULSKY, T.; SILVA, T. S.; MALUF, A. M.; SILVESTRE, M. S. F.; CHIEA, S. A. C.; CUSTÓDIO-FILHO, A.; MANTOVANI, W.; JUNG, S. L.; BARROS, F.; OLIVEIRA, L. C. A. Pteridófitas e fanerógamas. In: FIDALGO, O.; BONONI, V. L. R. (Ed.). Técnicas de coletas, preservação e herborização de material botânico - Série Documento. São Paulo: Instituto de Botânica, 1989. 62 p.

SILVA, M. P. Conhecimento etnobotânico de comunidades rurais da Serra de Campo Maior-PI, Brasil. 2010. $173 \mathrm{f}$. Dissertação (Mestrado em Desenvolvimento e Meio Ambiente) Universidade Federal do Piauí, Teresina. 2010.
SILVA, N. C. B. Bioprospeç̧ão de plantas medicinais com potencial biotecnológico: estudo etnofarmacológico de três comunidades tradicionais da região da Chapada Diamantina, BA. 2003. 227 f. Tese (Doutorado em Biotecnologia Vegetal) Universidade Federal do Rio de Janeiro, Rio de Janeiro. 2003.

SIMÕES, C. M. O.; MENTZ, L. A.; SCHENKEL, E. P.; IRGANG, B. E.; STEHMANN, R. J. Plantas da medicina popular no Rio grande do Sul. Porto Alegre: Ed. da UFRGS, 1989. 173 p.

SOUSA, R. S. Etnobotânica e etnozoologia de comunidades pesqueiras da Área de Proteção Ambiental (APA) do Delta do Parnaíba, nordeste do Brasil. 2010. 176 f. Dissertação (Mestrado em Desenvolvimento e Meio Ambiente) - Universidade Federal do Piauí, Teresina. 2010.

SOUSA, V. C; LORENZI, H. Botânica sistemática: guia ilustrado para identificação das famílias de Angiospermas da flora brasileira, baseado na APG II. Nova Odessa: Instituto Plantarum, 2005. 639 p.

SOUSA, V. C; LORENZI, H. Botânica sistemática: guia ilustrado para identificação das famílias de Fanerógamas nativas e exóticas no Brasil, baseado na APG III. Nova Odessa: Instituto Plantarum, 2012, 768 p.

STEFANINI, M. B.; RODRIGUES S. D.; MING, L. C. Ação de fitoreguladores no crescimento da erva-cidreira-brasileira. Horticultura Brasileira, Brasília, v. 20, n. 1, p. 18-23, 2002.

TEIXEIRA, S. A.; MELO, J. I. M. Plantas medicinais utilizadas no município de Jupi, Pernambuco, Brasil. Iheringia, Série Botânica, Porto Alegre, v. 61, n. 1-2, p. 5-11, 2006.

TOSTI, E.; COLLI, A. M. T. Estudo etnobotânico no município de Colômbia - SP. 2007. Disponível em: <http://www. unifafibe.com.br/revistasonline/arquivos/revistafafibeonline/ sumario/11/19042010102132.pdf >. Acesso em: 12 nov. 2013.

TROTER, R.; LOGAN, M. Informant consensus: a new approach for identifying potentialli effective medicinal plants. In: ETKIN, N. L. (Ed.). Indigenous medicine and diet: biobehavioural approaches. New York: Redgrave Bedford Hills, 1986. p. 91-112.

TULER, A. C. Levantamento etnobotânico na comunidade rural de São José da Figueira, Durandé, MG, Brasil. 2011. Disponível em: $\quad<$ http://www.cienciasbiologicas.alegre.ufes.br/sites/www. cienciasbiologicas.alegre.ufes.br/files/TCC\%20Am\%C3\%A9lia. pdf $>$. Acesso em: 5 jan. 2014.

WONG, J. L. G. The biometrics of non-timber forest product resource assessment: a review of current methodology. Roma: Europian Tropical Forest Research Network, 2000. 115 p. 